\title{
EXCOL: An EXtract-and-COmplete Layering Approach to Cartoon Animation Reusing
}

\author{
Lei Zhang Hua Huang Hongbo Fu
}

\begin{abstract}
We introduce the EXCOL method (EXtract-and-COmplete Layering) - a novel cartoon animation processing technique to convert a traditional animated cartoon video into multiple semantically meaningful layers. Our technique is inspired by vision-based layering techniques but focuses on shape cues in both the extraction and completion steps to reflect the unique characteristics of cartoon animation. For layer extraction, we define a novel similarity measure incorporating both shape and color of automatically segmented regions within individual frames and propagate a small set of user-specified layer labels among similar regions across frames. By clustering regions with the same labels, each frame is appropriately partitioned into different layers, with each layer containing semantically meaningful content. Then a warping-based approach is used to fill missing parts caused by occlusion within the extracted layers to achieve a complete representation. EXCOL provides a flexible way to effectively reuse traditional cartoon animations with only a small amount of user interaction. It is demonstrated that our EXCOL method is effective and robust, and the layered representation benefits a variety of applications in cartoon animation processing.
\end{abstract}

Index Terms — cartoon animation, layer extraction, layer completion, label propagation

\section{INTRODUCTION}

$\mathrm{C}$ Artoon animation, or animated cartoon, is a popular artistic illustration for storytelling. However, producing cartoon animations from scratch is a time-consuming task even for skillful artists. Given a large body of existing traditional animations, reusing them for helping generate novel animations has proved more productive [1], [2], [3].

In the modern production of cartoon animation, multiple layers are often employed: each layer corresponds to either a meaningful and complete foreground character, or panning background in which the character is moving. In drawing cartoons, the background is first painted as the "landscape", and then animated characters are superimposed on the background to form the final cartoon animation. However, such inherent layer information is lost when publishing the finalized cartoon animation, since the resulting cartoon video always appears as a single layer. Therefore, extracting layers from a cartoon video, i.e., the problem of cartoon animation layering, becomes crucial for fully reusing widely available cartoon animations and bringing historic cartoons into a more modern technological setting.

Cartoon animation layering, as part of an inverse problem of cartoon animation production, naturally demands two major tasks. First, every frame should be decomposed into disjoint regions, each of which represents a layer containing semantically meaningful content. The extracted layers are required to be coherent across frames. Apparently the number of layers needed varies with the complexity of underlying

- L. Zhang and H. Huang are with the Visual Media Computing Lab, Xi'an Jiaotong University. E-mail: cgzhanglei@gmail.com, huanghua@xjtu.edu.cn

- H. Fu is with the School of Creative Media, the City University of Hong Kong. E-mail: hongbofu@cityu.edu.hk scenes. Second, a layer completion step is further needed to fill missing parts caused by occlusion between layers. Although cartoon layering or segmentation is proved vital to effective reusing of classic cartoons, the existing works [1], [2], [4], [5] are limited to the problem of two-layer decomposition (i.e., to extract a foreground layer and a background layer) and do not deal with layer completion at all.

Both the problems of layer extraction and layer completion have been widely studied in the field of computer vision. Those vision-based techniques are commonly designed for general types of videos and typically use parametric motion models (e.g., based on an affine motion model [6]) for describing scene content within individual layers. However such motion models are generally not expressive enough for capturing highly exaggerated motions often exhibited in cartoon animations [1], making such vision-based techniques not suitable for cartoon animation layering.

We introduce a novel layering technique for cartoon animation, which we call EXCOL (EXtract-and-COmplete Layering). Noticing that cartoon animations, created with cellpainting or vector graphics tools, usually exhibit smooth and well-defined shapes, as well as large regions with simple colorization and visible outlines [4], [7], we fully explore shape cues extracted from an input cartoon video to facilitate both extraction and completion of layers (see an overview of our algorithm in Section 3). EXCOL is able to not only extract multiple semantically meaningful and temporally coherent layers in a single pass, but also faithfully recover structural features within possibly large missing regions in the extracted layers (see Figure 1 and the accompanying video). We have demonstrated that the resultant layers can be reused in a wide range of cartoon animation editing tasks, including recoloring, object replacement, reshuffling, and composition. 

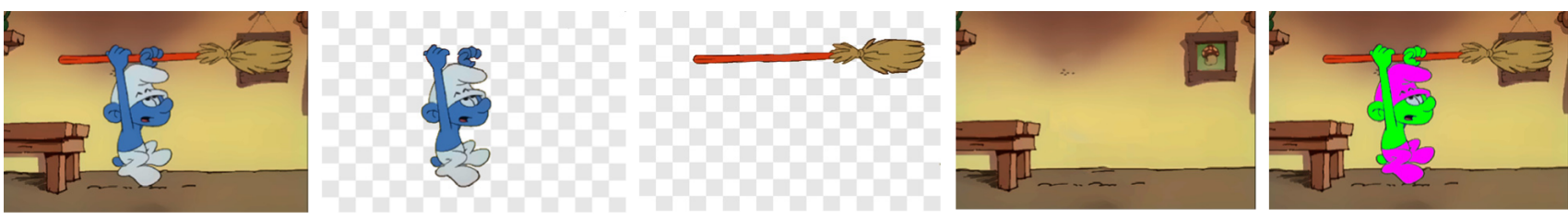

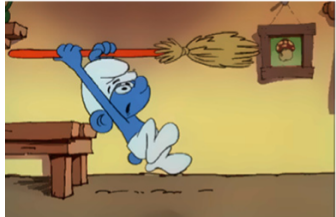

(a)

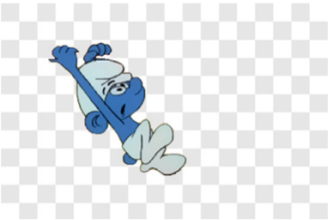

(b)

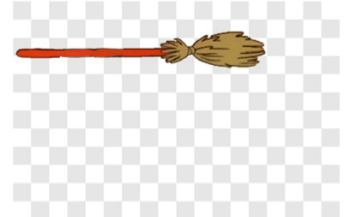

(c)

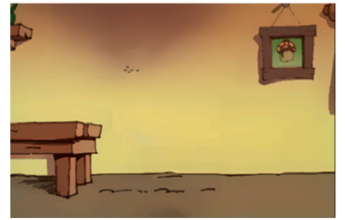

(d)

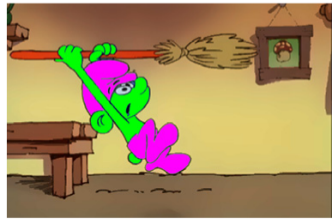

(e)

Fig. 1: Our extract-and-complete layering approach decomposes a cartoon animation (a) into semantically meaningful layers (b-d). The layered representation permits effective reuse of traditional animated cartoons and supports a variety of applications including cartoon recoloring (e). (Cartoon images are from The Smurfs.)

\section{Related Work}

Cartoon Animation Processing. Our work belongs to the scope of cartoon animation processing. There are many techniques published in the domain of cartoon processing, e.g., cartoon creation, displaying and editing [4], [5], [8]. But only few techniques are designed for reusing cartoon animations due to the challenging nature of the involved inverse problems. Bregler et al. [1] introduce a technique for reusing expressive cartoon motion styles by first capturing the motion from existing cartoon animations and then retargeting it to $2 \mathrm{D}$ or 3D characters. They extract cartoon regions of interest for motion capturing by simply analyzing their color distribution. While such simple segmentation might be sufficient for motion estimation, it often leads to an inaccurate layer representation especially for cartoon regions with complex color distributions, precluding applications like cartoon re-sequencing [2], [9]. de Juan and Bodenheimer [2] introduce a more accurate cartoon segmentation technique based on Support Vector Machines (SVM) classification. Their technique focuses on separating a single foreground character from the background and thus formulates the problem as a binary classification problem. Although it is mentioned in [2] that multiple cartoon layers can be possibly extracted by training a SVM classifier for each layer and applying individual classifiers in separate passes, this recursive layer extraction might cause accumulated segmentation errors especially for separating cartoon characters that are close to each other. In addition, neither the work of Bregler nor that of de Juan and Bodenheimer tackles the problem of cartoon layer completion.

There exist other solutions for two-layer decomposition of cartoon animation. For example, assuming a single foreground layer consisting of homogenous regions bounded by clearly visible outlines, Sýkora et al. [4] use outlines to segment black-and-white cartoons and then classify segmented regions by area thresholding. As we shall demonstrate later (e.g., in Figure 10), such simple area criterion might not already be robust for two-layer decomposition of modern cartoons with complex scenes, making its extension to multi-layer extraction rather challenging. Zhang et al. [5] present a two-layer extraction approach in their cartoon vectorization framework. Their approach focuses on extracting background pixels by examining their color variance in a registered panorama and simply regards the remaining pixels as those in the foreground layer. Therefore their approach is difficult to extend for extracting multiple foreground layers.

Video Layering. Video layering has been widely studied in the field of computer vision (see [10] for an insightful survey). Vision-based layering techniques typically assume that a scene can be approximately decomposed into several layers, each of which is consistently governed by a 2D motion model. Existing methods mainly vary with motion models (e.g., dense motion fields represented by optical flow [11], parametric motion models represented as affine transformations [6], [12], homography [10]) and motion segmentation algorithms for estimating motion model parameters (e.g., based on Kmeans [11], linear space [12], graph cut [6], [10], [13]). However, due to motion approximation errors, vision-based layering techniques often suffer from artifacts especially at regions near to layer boundaries. Since artifacts at cartoon contours are the most noticeable, such techniques are not suitable for cartoon layering. Recently, Wang et al. [14], [15] introduce a layering approach based on multi-label propagation, which is close in flavor to our approach. Their approach employs cumulative Gaussian Mixture Model (GMM) of pixel colors to determine the label conformity. The drawback of using only color information is its inaccuracy in classifying regions with similar color but on different layers, which is common in cartoons due to its simple colorization. Hence, for cartoon animation layering, more cues, like shape, should be incorporated to obtain more accurate layer extraction (see comparisons in Figure 13).

Most of vision-based layering algorithms attempt to minimize user interaction. Recently, several interactive object cutout techniques for videos [16], [17], [18] have been introduced into the community of computer graphics, achieving higher quality of layer extraction with more user interaction. We show that the unique characteristics of cartoon videos allow us to design a simple but effective technique for cartoon layering, with only a small amount of user interaction needed for typical cartoons. Finally, it is worth mentioning that as the 

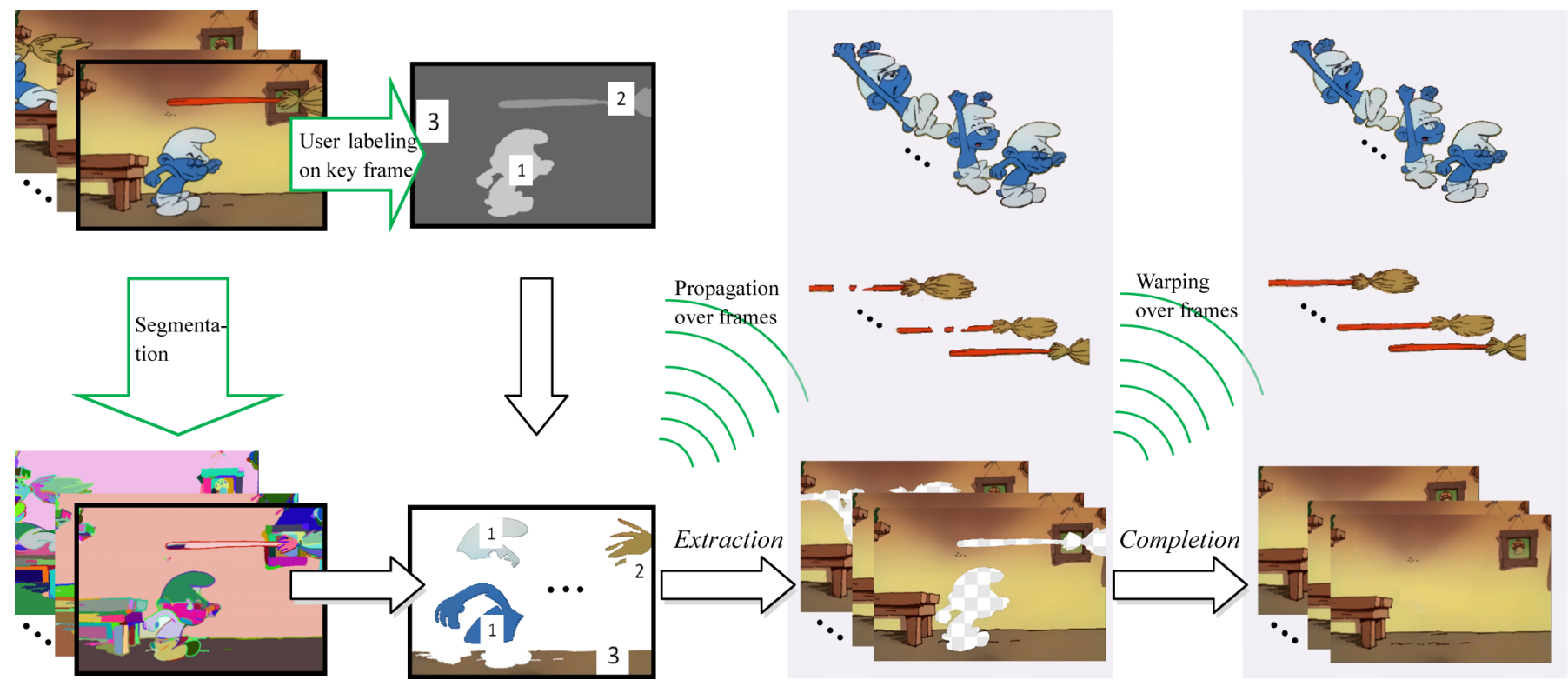

Fig. 2: Algorithm overview. Our EXCOL technique automatically propagates a small set of layer labels manually specified on a key frame (e.g., Label \#1 for the Smurf, the main character, Label \#2 for the broom and Label \#3 for the background) to pre-segmented patches of the other frames to extract temporally coherent layers. Missing regions in each layer (e.g., regions of the broom due to the occlusion by the hands of the Smurf, regions of the background due to the occlusion by the foreground objects) are then completed by a warping-based approach over the frames.

inverse problem of layer extraction, a local layering approach is recently proposed by McCann and Pollard [19] for easy composition of images.

Video Completion. Completion of general videos has also been an active research topic in recent years. Spatio-temporal coherence is an important issue when filling the "holes" in the video. Wexler et al. [20] extend the patch-based image completion approach [21] for video completion. A video sequence is treated as space-time volumes, and the missing areas are filled with the most similar space-time patches by optimizing a well-defined objective function. Jia et al. [22] introduce a fragment-based video completion approach, which shares the spirit of texture synthesis to fill missing parts by copying the most promising patches through frames. Jia et al. [23] present a video repairing approach that deals with background and foreground separately: the static background is completed using homography blending of layer segmentation while the foreground is completed by warping a sampled motion model from the sequences. Their approach is able to preserve temporal coherence after completion, but restricted to only videos complying with the adopted motion model.

\section{OVerview}

For simplicity, we assume that an input cartoon video contains a fixed number of layers across all frames, denoted by $N$ (e.g., $N=3$ for the example in Figure 2). Our problem of cartoon animation layering can be formally defined as follows. Given a sequence of cartoon video frames $\left\{I_{k}\right\}$, our goal is to first decompose each frame $I_{k}$ into $N$ disjoint regions (layers) $I_{k}=\bigcup\left\{L_{k}^{r}, 1 \leq r \leq N\right\}$, with each layer assigned a label $l_{r}$ to indicate its layer index, and then complete missing areas in the layers to obtain a full-formed representation over the frames $\mathscr{L}=\left\{L^{r}\right\}$. For simple exposition, we let $L^{N}$ denote the background layer and $L^{r}, 1 \leq r \leq N-1$, the animated object layers. The work flow of our layering approach is shown in Figure 2 and described below.

Layer Extraction. Observing that cartoon animations often exhibit easily detectable patches, we extract layers by labeling pre-segmented patches of each frame (Section 4), instead of operating at the pixel level, as common in visionbased layering techniques. The user initiates the extraction by interactively marking desired layers in a key frame, with each layer automatically assigned a unique layer label. For example, three layers are marked in the example of Figure 2: two for foreground objects and one for the background. The layer labels are then automatically propagated to the rest of the frames by optimizing a multi-label system, which permits extraction of multiple layers in a single pass (Section 4.1). To ensure consistent labeling of same objects across different frames, we define a novel similarity measure incorporating both the shape and color information of patches. After label propagation, patches are grouped by label to form layers, reflecting the same set of semantically meaningful objects marked on the key frame by the user. Finally each layer is refined by finding its associated decorative lines (Section 4.2).

Layer Completion. Our next step is to detect and fill missing regions of individual layers due to occlusions between the overlapping layers. A warping-based approach is adopted for this task, since cartoon shapes in each layer usually undergo regular transformation over frames (Section 5). Given the different motion natures of background and object layers, we use different warping transformations to align and complete them: a homography transformation for background layer (Section 5.1) and global-to-local transformations for moving object layers (Section 5.2). Since the completion is done 
through background and object registration, our approach allows the recovery of structural features in a temporally coherent manner.

\section{LAYER EXTRACTION}

As we intend to extract semantically meaningful layers, a small amount of user interaction is necessary to guide layer extraction. Like most of interactive video segmentation techniques (e.g., [6], [18]), we let the user provide guidance on some frame, which we call a key frame. The key frame, denoted as $k_{0}$, is typically the frame where desired foreground layers have little overlapping, which ease the layer completion step, as we will show later. The user is required to decompose the key frame into multiple disjoint regions, with each region representing a meaningful layer with a unique layer label (see Figure 2). Such decomposition can be easily achieved by interactive image segmentation methods such as lazy snapping [24], grabcut [25] or by employing cartoon painting tools, like LazyBrush [26]. Such user guidance supplies two instructions for the subsequent automatic label propagation process: desired content within individual layers and the number of layers over the frames (i.e., $N$ ). Let $\left\{L_{k_{0}}^{r}, 1 \leq r \leq N\right\}$ denote semi-automatically extracted layers in the key frame, where each layer $L_{k_{0}}^{r}$ corresponds to a planar region associated with a label $l_{r}$. Our next step is to propagate these guidance layer labels over frames to obtain the layers in the other frames.

It has been observed that cartoon animation is typically composed of large colored regions with regular shape and narrow decorative lines [5], [7] (see Figure 3). Since decorative lines are generally thin yet with finite width, we choose to first propagate labels over regions and then decorate labeled regions with decorative lines. We use the trapped-ball approach [5] to segment all the frames before label propagation, which is particularly suitable for cartoon segmentation. For brevity, in the following discussion we simply refer patches as the segmented large regions, and lines as the decorative lines. Note that the key frame is also segmented, possibly resulting in some patches with the same labels (Figure 2).

\subsection{Label Propagation on Patches}

The label propagation from the key frame to the other frames can be done either back or forth. For brevity, we only explain how to propagate the labels from the key frame to the frames after it. Let frame $I_{k}$ be the one which has been labeled (i.e., starting from $I_{k_{0}}$ ) and $I_{k+1}$ the frame to be labeled. The key idea to assign a label $l_{i}$ to segmented patch $S_{i} \in I_{k+1}$ is to compare $S_{i}$ with the patches in frame $I_{k}$, and assign to $S_{i}$ the label with the most similarity in frame $I_{k}$. Hence, the heart is a well-defined similarity measure between two segmented patches.

It is observed as the natural features of cartoons [2] that segmented patches typically preserve their color and shape in the consecutive frames. For example, as shown in Figure 2, the color and shape of the Smurf's body change only slightly between two successive frames. Hence, the similarity between two patches can be defined considering their color and shape properties. In fact, these features have already been exploited

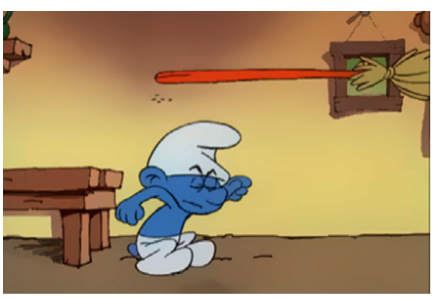

(a)

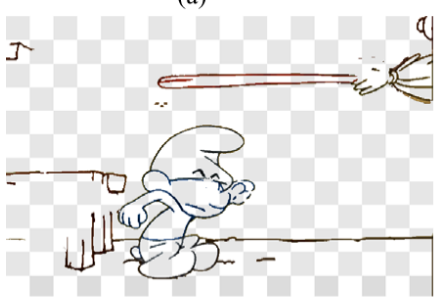

(c)

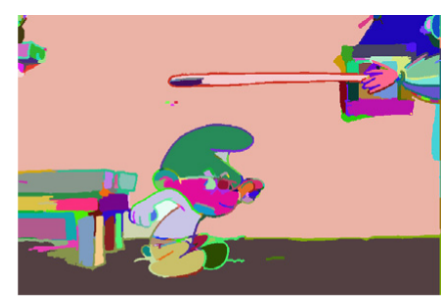

(b)

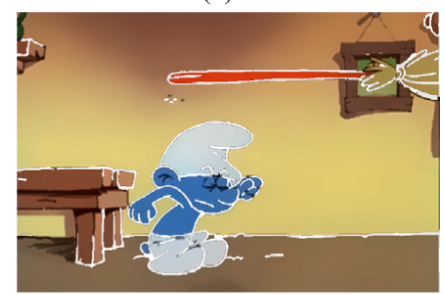

(d)
Fig. 3: Segmentation of a cartoon image (a) by the trapped-ball approach generates narrow decorative lines (c) and large colored patches (d). Decorative lines usually surround large patches. (b) shows the segmented regions, with different segments in different colors.

in some previous segmentation methods for cartoon videos, e.g., using probabilistic matting [1] and learning based classifier [2]. However, as described in [2], their method would result in outliers with erroneous label after segmentation, because the regional compactness is ignored in the binary classification. To get more accurate layer extraction, we use the segmentation scheme of graph cut family [27], which integrates region, boundary and shape properties of segments and better suits our extraction requirement.

To enable graph cuts segmentation setting, we first define the similarity measure based on color and shape properties. As segmented patches have roughly uniform color distribution, it is sufficient to use the difference in their average color to define color similarity. There are many shape descriptors to depict the profile of patch shape. Here we adopt shape context as the shape descriptor because of its robustness and invariance under transformations of translation, uniform scaling and rotation [28]. More specifically, the similarity measure between two patches $S_{i}$ and $S_{j}$ is defined as

$$
M\left(S_{i}, S_{j}\right)=\left\|C_{i}-C_{j}\right\|+\lambda H\left(S_{i}, S_{j}\right)
$$

where $C_{*}$ is the average color of patch $S_{*}, H$ is the shape matching cost based on shape context [28], and $\lambda$ is the weight to control shape and color tradeoff according to the segmentation results.

Once the similarity measure is defined, label propagation can be cast as a multi-label problem, typically modeled in Markov Random Field (MRF) frameworks. In this multi-label problem setting, each frame $I_{k}$ is modeled as a graph $\mathscr{G}_{k}=$ $\left\{\mathscr{V}_{k}, \mathscr{E}_{k}\right\}$, where the nodes $\mathscr{V}_{k}=\left\{v_{1}, \ldots, v_{n_{k}}\right\}$ correspond to the set of patches, and the edges $\mathscr{E}_{k}=\left\{\left\langle v_{p}, v_{q}\right\rangle\right\}$ denote the connectivity of patches. That is, if patches $v_{p}$ and $v_{q}$ share a decorative line, there is an edge $\left\langle v_{p}, v_{q}\right\rangle$ in $\mathscr{G}_{k}$ (see Figure 4). Then the optimal labeling on $I_{k+1}$ is found by minimizing the 

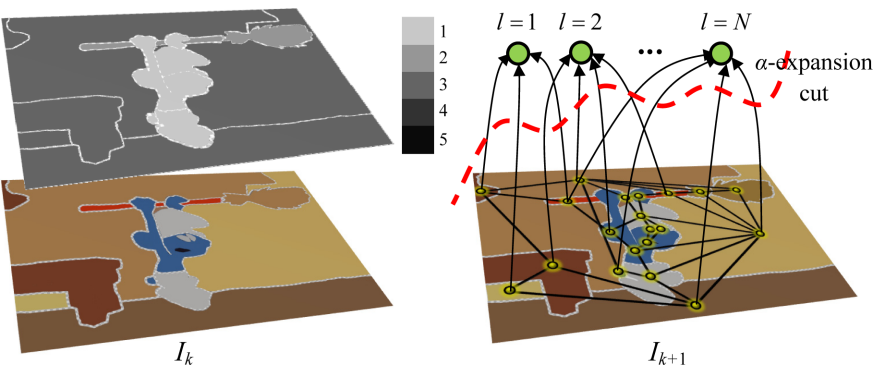

Fig. 4: Label propagation is obtained by tracing the color and shape properties of patches between consecutive frames. Left: Segmented patches in frame $I_{k}$ and its corresponding labels, indicated by gray scale mask. Right: The optimal labeling in the current frame $I_{k+1}$ is obtained using $\alpha$-expansion graph cuts defined on the patches.

following energy function:

$$
E_{k+1}(l)=\sum_{i} D_{i}\left(l_{i}\right)+\sum_{<p, q>\in \mathscr{N}} V_{<p, q>}\left(l_{p}, l_{q}\right)
$$

where $i$ traverses all the nodes $\mathscr{V}$ and $\mathscr{N}$ is the neighborhood relationship defined by $\mathscr{E}$. The energy function (2) is the classical representation of a posterior energy in MRF frameworks [29], and thus can be optimized via graph cuts for the well-defined data item $D_{i}$ and smooth item $V_{<p, q>}$, which are be introduced below.

The data item $D_{i}\left(l_{i}\right)$ measures the conformity of patch $S_{i}$ labeled to the layer $l_{i}$. For each patch $S_{i} \in I_{k+1}$, we compute the label conformity to layer $l_{i}$ based on the similarity measure as $d_{i}^{l_{i}}=\min \left\{M\left(S_{i}, S_{j}\right) \mid S_{j} \in L_{k}^{l_{i}}\right\}$. Therefore, the data item $D_{i}$ is defined as

$$
D_{i}\left(l_{i}\right)=\frac{d_{i}^{l_{i}}}{\sum_{j=1}^{N} d_{i}^{l_{j}}} .
$$

The smooth item $V_{<p, q>}\left(l_{p}, l_{q}\right)$ measures the cost of assigning label $l_{p}$ and $l_{q}$ to the adjacent nodes $v_{p}$ and $v_{q}$, which imposes the spatial smoothness of labels. Formally, $V_{<p, q>}$ is defined as

$$
V_{<p, q>}\left(l_{p}, l_{q}\right)=\frac{1}{1+\left\|C_{p}-C_{q}\right\|}\left\|l_{p}-l_{q}\right\|,
$$

where $C$ is the average color of segmented patch $S$ in the frame $I_{k+1}$.

Solving the above multi-label problem of Equation (2) is NP-complete. Thus we use the $\alpha$-expansion graph cuts [30] to iteratively find the approximate optimization. Then, the label results in frame $I_{k+1}$ can be obtained. From these labels, patches on $I_{k+1}$ are classified into the corresponding layers to achieve a coarse layers extraction.

Discussions. Similar energy functions to Equations (2) (4) have also been used in lazy snapping [24] for image segmentation. Lazy snapping uses only colors as main cue to compute the likelihood to foreground (background) in a single image; while in our cartoon animation layering setting, both color and shape are considered to compute the similarity, and confident "foreground (background)" comes from the layers in the previous frame.

\subsection{Layer Refinement on Lines}

Our previous label propagation step results in a coarse layer extraction, i.e., labeled patches without decorative lines associated (Figure 5 (top-left)). Next we need to label the decorative lines to get the accurate extracted layers. Decorative lines usually surround a character and thus can be reached by expanding the regions of the labeled patches with a moderate range $w$, where $w$ is the width of decorative lines (Figure 5). Noted that $w$ is variant but its value can be directly obtained through the trapped-ball segmentation approach [5], measured as the distance between pairs of edges detected by traditional Canny operator.

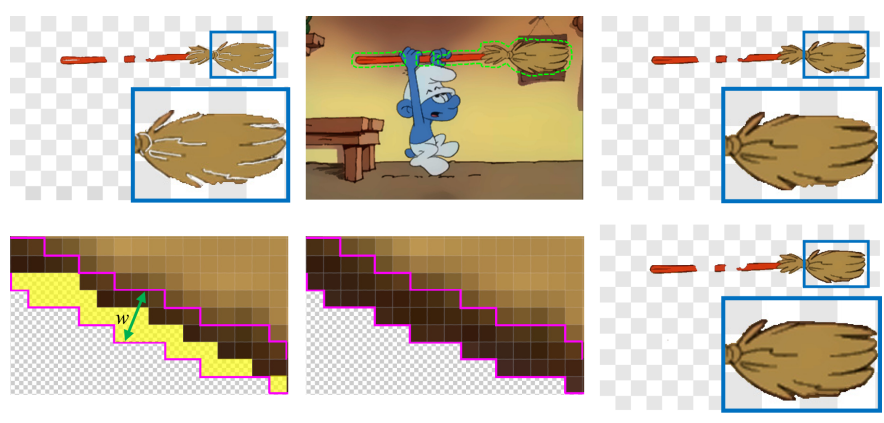

Fig. 5: Top: Grabcut refinement. From left to right: before refinement, regions to be refined (enclosed by dash green lines), after refinement. Bottom: Laplacian diffusion. From left to right: before diffusion (decorative lines in purple and mislabeled pixels in yellow), after diffusion, and final result.

As the initial colors in narrow regions for the decorative lines are often mixed by the colors from adjacent layers, which is typically the case for cartoons of low resolution, we take a two-step procedure: Grabcut refinement for extracting desired colors and Laplacian diffusion for filling the regions originally occupied with undesired colors. In each frame, the decorative lines will be labeled layer by layer from $L^{1}$ to $L^{N}$. If layer $L^{r}$ is associated with decorative lines (this can be inferred from user interaction in the key frame), then the regions belonging to $L^{r}$ are expanded by dilating them $2 w$ range around (Figure 5), which ensures the decorative lines are covered. Then, setting the patches on this layer as foreground seed regions, Grabcut [25] is employed to further segment the frame, which extracts desired colors in the regions for the decorative lines (Figure 5 (bottom-left)). However, Grabcut might lead to jaggy boundaries due to the fuzzy resolution near the decorative lines, thus possibly resulting in mislabeled pixels in the decorative line region (Figure 5 (bottom)). To address the problem we use a simple Laplacian diffusion scheme to further improve the boundary smoothness. Specifically, for each mislabeled pixel $p$, its color $c_{p}$ is recomputed as the average of its 4-connected neighbors $\mathscr{N}_{p}$ in the decorative line region, i.e., $c_{p}=\sum_{q \in \mathscr{N}_{p}} c_{q} /\left|\mathscr{N}_{p}\right|$. The diffusion can be iteratively performed for all the mislabeled pixels until color change is below a threshold. In our implementation, usually 3 iterations are enough to achieve smooth boundaries.

After the label propagation and layer refinement, we get a layered representation of cartoon animation over the frames. User interaction is allowed to adjust the layer labels on the 

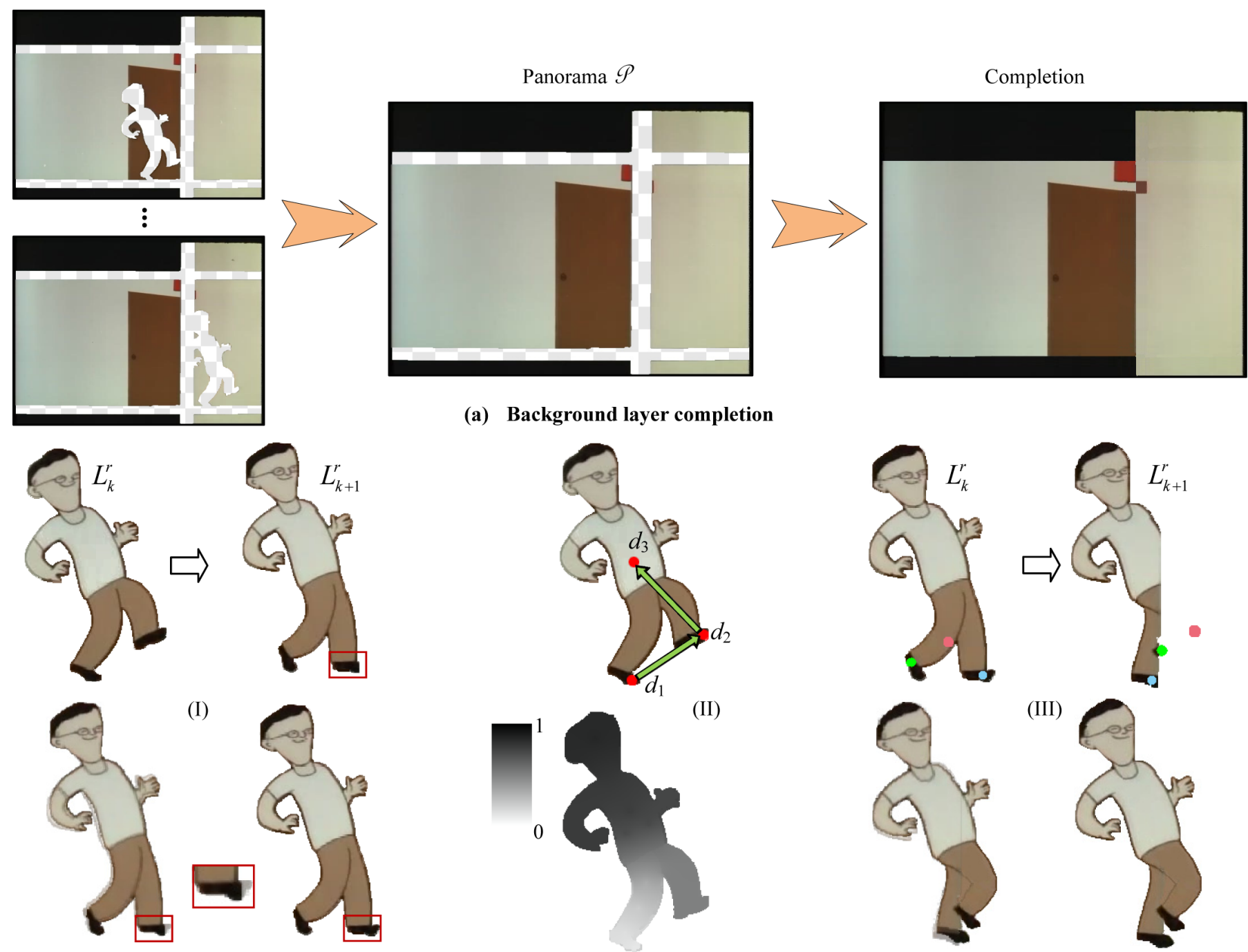

(a) Background layer completion

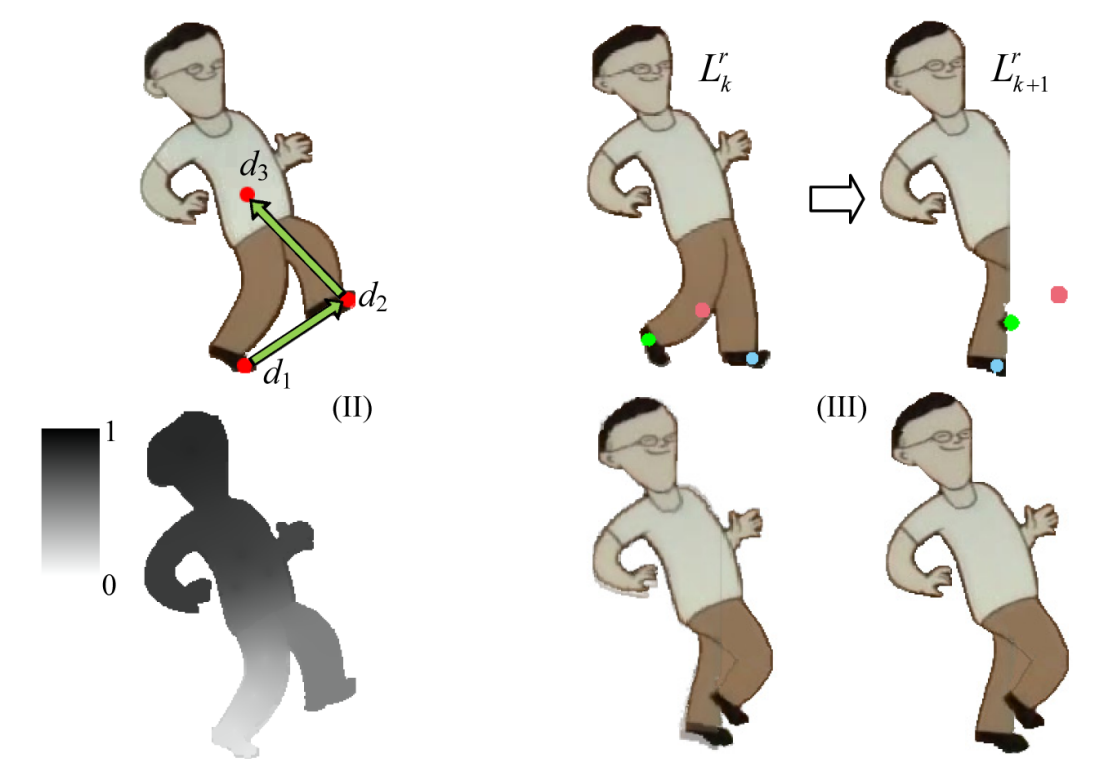

(b) Foreground layer completion

Fig. 6: (a) Background patches from different frames are first registered to a single panorama, possibly with "holes" due to the occlusion of foreground layers; then the holes are filled by an image completion approach [21]; finally the completed background in individual frames is retrieved back from the completed panorama. (b) Missing parts on a foreground layer are filled by warping the completed layer $L_{k}^{r}$ at frame $k$ to the corresponding occluded layer $L_{k+1}^{r}$ at frame $k+1$. This can be done automatically for small missing regions like the example in (I). Whereas in the presence of severe self-occlusion or large deformation (III), a small amount of user interaction is needed for specifying correspondence constraints (e.g., pairs of color dots in III) and/or specifying inequalities for depth calculation (II) on the key frame (e.g., green arrows indicating depth order: $d_{1} \leq d_{2} \leq d_{3}$ ). (Cartoon images are from The New Door Bell.)

regions if mistakenly labeled (see examples in the accompanying video). Our next step is complete possible holes in each layer caused by occlusion to get a semantically full-formed layer representation.

\section{Layer Completion}

The production process of cartoon animation gives us hints to layer completion. In drawing cartoons, usually the background, where the characters are moving, is first made into a large-size panorama, so-called "pan background". Then, each frame is produced with the camera following the characters as they move across the background, whereas changes of characters between two successive frames are often not significant. Inspired by these observations, we use a warping-based approach to complete the background and object layers in a coherent way. Since the motions of background and foreground objects are of very different degrees of freedom, we use different transformations to model the warping for background and foreground.

\subsection{Completion of Background Layer}

After separating foreground characters or objects from the background, the background layer $L_{k}^{N}$ of each frame often exhibits holes or missing areas, as shown in Figure 6 (a). It is important to achieve a spatio-temporally coherent layer completion for background, because static background usually makes any possible flickering artifacts look more apparent during frame transition. Since the background in each frame is typically cast from a panoramic image, we first reconstruct that panorama from all the background patches in individual frames. 
Constructing a panoramic background image essentially requires locating and registering the background layer $L_{k}^{N}$ of every frame in a single global coordinate system [4]. Each frame corresponds to a warped region in the global system by a homography transformation $H_{k}: L_{k}^{N} \rightarrow \tilde{L}_{k}^{N}$. By registering all the frames through respective warping transformations, we obtain a panoramic image $\mathscr{P}$ (see Figure 6 (a)), possibly with regions yet missing in the panorama. Either area-based or feature-based image registration approaches [31] can be employed to construct the panoramic background. In our implementation, we adopt a feature-based approach: we rely on SIFT [32] to compute the homography transformations, since it is invariant under translation, rotation and scaling, thus better dealing with cartoons under different background motion, e.g., camera zooming or translation (Figure 16). As common in feature-based approaches, we further resort to RANSAC [33] to filter out outliers and thus get more stable registration results.

If there still exist missing regions in the panorama $\mathscr{P}$, an image completion approach proposed by Drori et al. [21] is employed to fill the missing regions to obtain a complete panorama background (see Figure 6). Finally we retrieve the complete background layer in each frame by using the inverse homography $H_{k}^{-1}$. Since these completed layers come from one single image (i.e., the completed panorama), there is absolutely no flickering during the transition, guaranteeing temporal coherence on the background layers over the frames, even for examples with moving background (e.g., Figure 16 (b)).

\subsection{Completion of Foreground Layers}

Unlike the background layer, whose visual movement is typically due to camera motion of low degree of freedom, foreground characters or objects often exhibit much more complex motion that is possibly arbitrary. To make things worse, there possibly exists severe self-occlusion on the same layer, e.g., legs in Figure 6 (b-III), which makes completion of foreground layers rather challenging. Inspired by recent works on cartoon image registration [8] and depth reconstruction [34], we propose a global-to-local warping approach to sequentially warp a completed foreground layer $L_{k}^{r}$ at frame $k$ to help complete the corresponding layer $L_{k+1}^{r}$ in the next frame, in which auxiliary depth information can be optionally combined to guide the correct warping transformation.

We start with the key frame $k_{0}$, which has been chosen to have little overlapping on the foreground layers, as mentioned in Section 4. In other words, $L_{k_{0}}^{r}$ is typically complete or has very small holes which can be easily filled. Assuming the layer $L_{k}^{r}$ has been completed to a full-formed representation, we now consider the completion of layer $L_{k+1}^{r}$ in the next frame. The core of completing $L_{k+1}^{r}$ is to find a proper warping transformation $T_{k, k+1}$ that best aligns $L_{k}^{r}$ to $L_{k+1}^{r}$.

The ideas of as-rigid-as-possible (ARAP) cartoon registration [8] are borrowed here to compute the best warping $T_{k, k+1}$. The ARAP approach applies Push and Regularize steps iteratively to find the best matching from a source image to a target image. ARAP works well for articulated shapes even with notable changes in appearance. We found that the original ARAP approach is able to successfully complete layers with small missing regions (e.g., the occluded part on the shoes in Figure 6 (b-I)). However, it performs poorly for the case of severe occlusion (e.g., Figure 7 (c)). This is because there have two distinctions in the settings between our layer completion and the original ARAP approach: i) unlike ARAP where source and target images both have complete shapes, the shape of layer $L_{k+1}^{r}$ in our case is incomplete due to the occlusion by the other layers (see Figure 6 (b)); ii) in contrast to global optimal registration, layer completion focuses on the warping in the local regions where the occlusion occurs.

Considering these two distinctions, we propose to adapt ARAP image registration in a global-to-local manner to make it more suitable to our layer completion phase. We also introduce easy-to-use user interfaces to greatly improve completion results but with little user interaction. Similar to [8], we embed the complete layer $L_{k}^{r}$ into an adaptive regular square lattice $\left\{p_{i}\right\}$ (see Figure 7 (a))

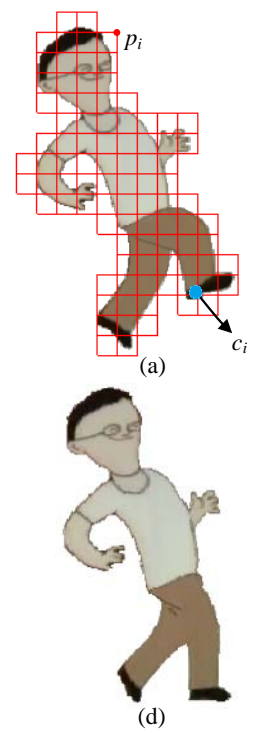

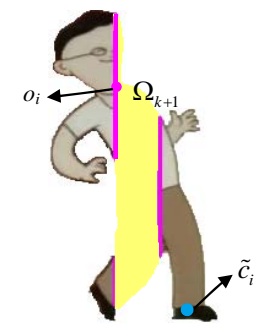

(b)

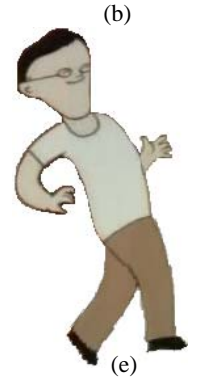

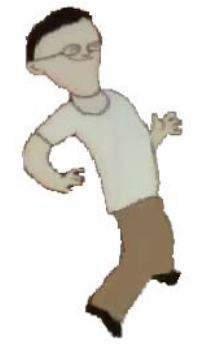

(c)

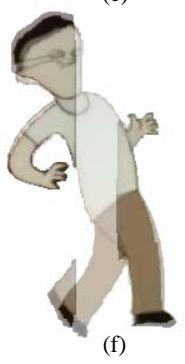

Fig. 7: Global ARAP warping with constraints. (a) Source foreground object $L_{k}^{r}$ is embedded in a square lattice $\left\{p_{i}\right\}$. (b) Target foreground object $L_{k+1}^{r}$ is partially occluded by layers in front, with missing regions (in yellow) denoted as $\Omega_{k+1}$ and their boundary points denoted as $\left\{o_{i}\right\}$. (c) Warped version of $L_{k}^{r}$ by the original ARAP approach. (d) Global ARAP warping with correspondence constraints (i.e., $\left\{\left(c_{i}, \tilde{c}_{i}\right)\right\}$ ) but without auxiliary depth. (e) Global ARAP warping with both correspondence and depth constraints. (f) Overlapping of $L_{k+1}^{r}$ and warped $L_{k}^{r}$ (e) shows that global ARAP warping alone might still not be sufficient for perfect completion.

\subsubsection{Global ARAP Warping with Constraints}

We observed that the original ARAP approach might cause improper shift vectors in the Push step of ARAP (e.g., Figure 7 (c)), since it is completely unsupervised. This problem can be solved by manually specifying a small set of pairwise position constraints $\left\{\left(c_{i}, \tilde{c}_{i}\right) \mid c_{i} \in L_{k}^{r}, \tilde{c}_{i} \in L_{k+1}^{r}, i=1 \ldots C\right\}$ on the two layers (see colored points in Figure 6 (b-III) and Figure 7). Then, the warped shape of $L_{k}^{r}$ with the user-specified 
constraints is obtained by minimizing:

$\left\{\tilde{p}_{i}\right\}=\underset{\tilde{p}_{i}, \mathbf{R}_{s}, \mathbf{t}_{s}}{\operatorname{argmin}} \sum_{s=1}^{S}\left\|\tilde{p}_{i, s}-p_{i, s} \cdot \mathbf{R}_{s}-\mathbf{t}_{s}\right\|^{2}+\lambda \sum_{i=1}^{C}\left\|\tilde{c}_{i}-c_{i} \cdot \mathbf{R}_{j}-\mathbf{t}_{j}\right\|^{2}$,

where $\left\{\tilde{p}_{i}\right\}$ are the positions of the warped lattices points (subscript $s$ indicating which square it belongs to), $\mathbf{R}_{s}$ is a rotation matrix, $\mathbf{t}_{s}$ is a translation vector and $S$ is the number of lattice squares.

The energy function (5) is the same as that described in [8] except for the second term for our user-specified constraints. Please refer to [8] for the algorithm details of the first energy term. To solve this minimization problem we borrow the iterative ARAP solver but take the constraints into account: push lattice points to the optimal positions defined by both color difference [8] and the constraints; and regularize lattices to obtain shape consistency. Finally, the global warping transformation from $L_{k}^{r}$ to $L_{k+1}^{r}$ is calculated as

$$
T_{k, k+1}\left(p_{i}\right)=\tilde{p}_{i},
$$

which warps the complete layer $L_{k}^{r}$ to the shape $\tilde{L}_{k+1}^{r}=$ $T_{k, k+1}\left(L_{k}^{r}\right)$.

In the presence of self-occlusion on layer $L^{r}$ over the frames, extra user interaction is needed to add auxiliary depth. Otherwise, artifacts like that shown in Figure 7 (d)) would appear. The depth can be added by using the technique proposed by Sýkora et al. [34] in the key frame. But instead of setting (in)equalities over all the patches, we only need to specify inequalities on the self-occlusion regions. For the example in Figure 6 (b-II), only two inequalities (green arrows) are sufficient to obtain necessary depth for warping layers. The depth will be subsequently propagated to the other frames following the warping transformation. Because the depth information of $L_{k}^{r}$ is available, we can get the correct appearance of $\tilde{L}_{k+1}^{r}$ for completion (see Figure 7 (e)).

Generally, $\tilde{L}_{k+1}^{r}$ can well fit the unoccluded regions on layer $L_{k+1}^{r}$, and the warped part corresponding to occlusion can be used to fill its missing regions. However, since $T_{k, k+1}$ is globally computed by regularizing all the lattice squares, matching results within the occlusion regions might not be optimal for layer completion (see Figure 7 (f)). Hence, we next perform a local ARAP warping to refine the transformation near the occluded regions to achieve more precise completion.

\subsubsection{Local ARAP Warping Refinement}

To refine the warping, we need to first identify the occluded regions on $L_{k+1}^{r}$. It is known that automatic detection of occlusion between layers is a challenging task on its own in computer vision [10]. To simplify the problem, we let the user indicate occlusion order of layers marked for the key frame (Section 4) and assume that the occlusion order keeps unchanged over frames. More specifically, it is assumed that layer $L_{k}^{r}$ will not be partially or completely occluded by $L_{k}^{t}$ when $t \geq r$. With such assumption, it is easy to identify part of the boundaries of the occluded regions in $L_{k+1}^{r}$ as the intersection boundaries between $L_{k+1}^{r}$ and layers $L_{k+1}^{t}, t<r$ (see the pink lines in Figure 7 (b) or 8 (a)). Let $O=\left\{o_{i}\right\}$ denote the points along such boundaries.
The key idea of local ARAP warping is to use the segmented patches of $L_{k+1}^{r}$ near to the occluded regions to locally drive the (further) warping of $\tilde{L}_{k+1}^{r}$. Let $V_{k+1} \subset L_{k+1}^{r}$ be the set of such patches (including the decorative lines), each of which contains $O$ as part of its boundaries (Figure 8 (a)). Denote $B_{k+1}=\left\{b_{k+1, i} \mid b_{k+1, i} \in \partial V_{k+1}\right.$ and $\left.b_{k+1, i} \in \partial L_{k+1}^{r}\right\}$ as the points which are along both the boundaries of $V_{k+1}$ and $L_{k+1}^{r}$ but excluding $O$. Next for each $b_{k+1, i}$, we find its closest point $\tilde{b}_{k+1, i} \in \partial \tilde{L}_{k+1}^{r}$ (Figure 8 (b)) and then use Equation (5) to warp $\tilde{L}_{k+1}^{r}$ with pairs of corresponding points $\left\{\left(\tilde{b}_{k+1, i}, b_{k+1, i}\right)\right\}$ as the new set of constraints $C$. These steps are iteratively performed, forming an ICP-like (iterative closed point) approach [35]. We stop the iteration until the distance between $\left\{b_{k+1, i}\right\}$ and $\left\{\tilde{b}_{k+1, i}\right\}$ is below a given threshold (Figure 8 (c)).

After local ARAP warping refinement is performed, we obtain the final warped layer $\tilde{L}_{k+1}^{r}$, which can be seen as an incarnation of $L_{k+1}^{r}$ in the next frame. Then, the missing parts on $L_{k+1}^{r}$, denoted as $\Omega_{k+1}$, can be easily identified as the regions of $\widetilde{L}_{k+1}^{r}$ that are bounded by the intersection boundaries between $L_{k+1}^{r}$ and $L_{k+1}^{t}(t<r)$ (see Figure 7 (b)). Finally transplanting the content within $\Omega_{k+1}$ from $\tilde{L}_{k+1}^{r}$ to $L_{k+1}^{r}$ fills the missing regions in $L_{k+1}^{r}$ (Figure 6 (b) and Figure 8 (d)).

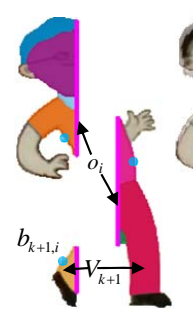

(a)

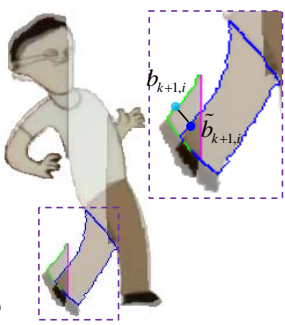

(b)

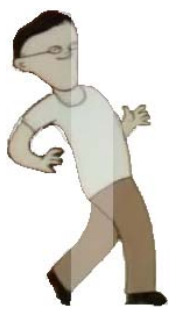

(c)

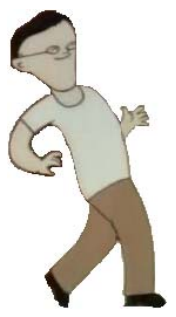

(d)
Fig. 8: Local ARAP warping to refine foreground layer completion. (a) Segmented patches (colored) near to the occluded regions, denoted as $V_{k+1}$. (b) For each boundary point $b_{k+1, i}$ of $V_{k+1}$, the closest point $\tilde{b}_{k+1, i}$ is found on the boundary of the transformed layer after global ARAP warping. (c) Such pairs of correspondence points drive local ARAP warping of $\tilde{L}_{k+1}^{r}$. (d) The final completion result.

\section{Results and Applications}

We have tested our layering approach on a variety of cartoon animation. See some of the layering results in Figures 1 and 9. These layering results can be further used to do various cartoon animation processing (see examples in Section 6.1). The accompanying video exhibits the final layering and processing results.

Timings. For every example, our approach needs a small amount of user interaction for initiating guidance layer labels in the key frame. Then, EXCOL can run automatically to produce the desirable layers. The computational time required for layer extraction and completion depends on a variety of factors, including the number of layers, frames and the segmented patches and lines. The numbers in Figure 9 show the typical timings for the main steps of EXCOL in one frame, measured on a $3 \mathrm{GHz} \mathrm{PC}$ with $3 \mathrm{G}$ RAM. There are two cases where additional user interaction is needed to produce more accurate results. First, if there are incorrect 


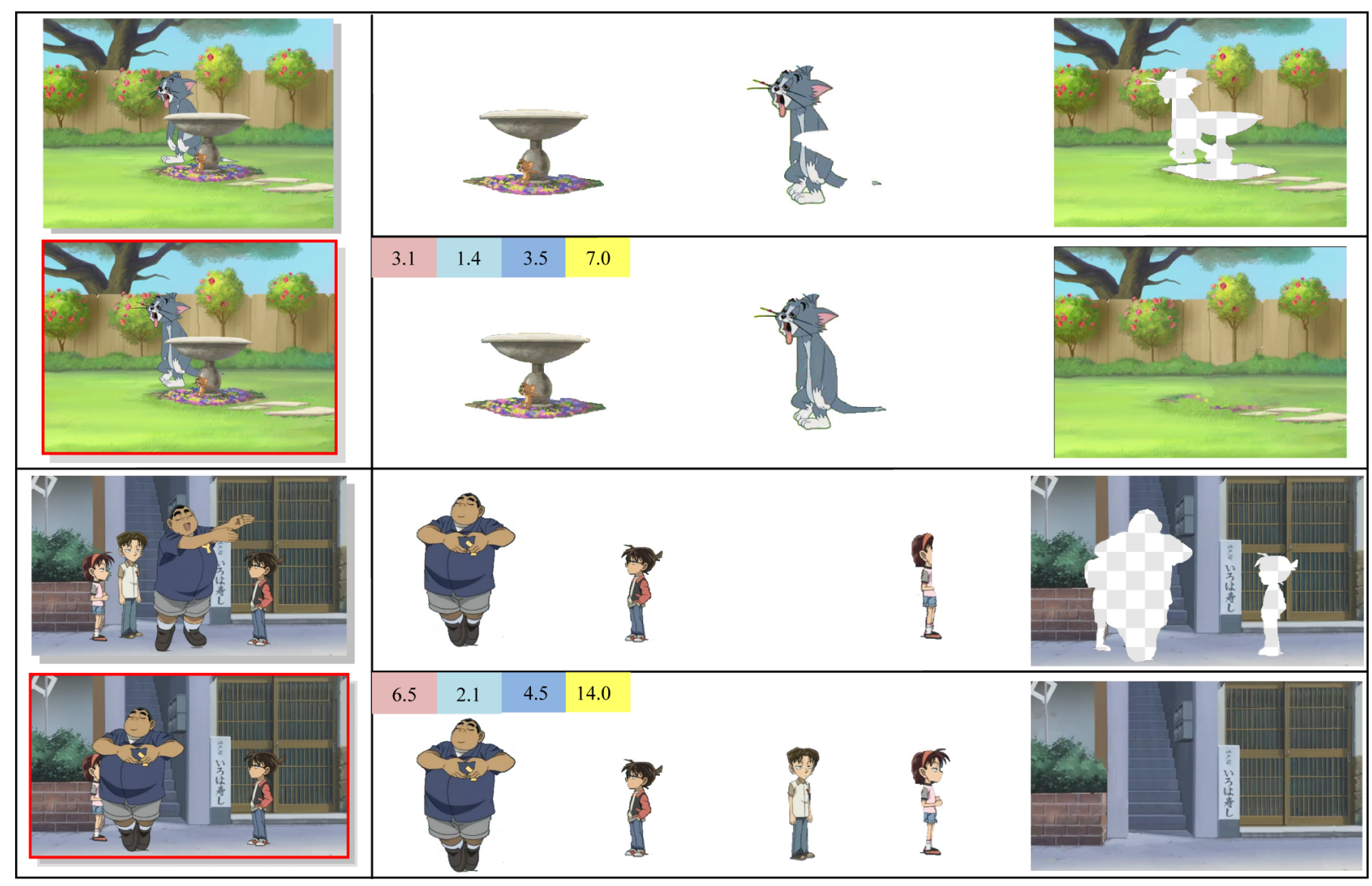

Fig. 9: Examples of extracted layers and completed layers corresponding to input frames highlighted with red rectangle, whose previous frames are shown as well for reference. The numbers in color denote the timing (in seconds) for label propagation (red), layer refinement (green), background completion (blue) and foreground completion (yellow) on the corresponding frames. (Cartoon images are from Tom and Jerry (top) and Detective Conan (bottom).)

labeling regions during label propagation, a scribble-type user interface like the one used in LazyBrush [26] is adopted to produce more accurate layer extraction with little sacrifice. Second, in the presence of self-occlusion or large occlusion on foreground layers, a small amount of user interaction is needed to add auxiliary depth information and constraints for layer completion. Please see the interactive sessions in the accompanying video.

Comparisons. We have compared EXCOL with representatives of previous cartoon processing techniques involving layer extraction and completion. Figure 10 shows some layer extraction and completion results with the approaches used in colorization of black-and-white (CBW) [4] and vectorizing cartoon animation (VCA) [5]. Unlike our EXCOL , which is able to accurately extract multiple layers, CBW and VCA are designed for two-layer decomposition. It can be seen that CBW is not reliable even for the case of two-layer extraction due to its adopted simple area-based classification criterion. Although VCA is more robust than CBW to extract the background layer, as discussed in Section 2, it is unclear how to extend VCA for multi-layer extraction. In both CBW and $\mathrm{VCA}$, the problem of layer completion is studied only for background layers.

Another potential solution of layer extraction is to propagate
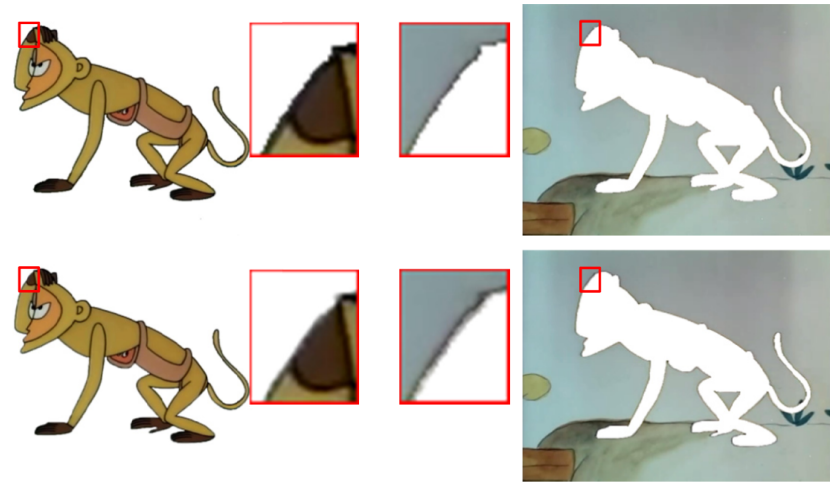

Fig. 11: Layer boundaries obtained in the layer extraction step using EXCOL (top) and LazyBrush [26] (bottom).

labels according to structural similarity prediction, which has been successfully applied in auto-painting of cartoons [4], [8], [26]. From an initial labeling indexed with color palette or strokes in the key frame, labels are delivered to the regions with best structural similarity in the next frame by using either patch-pasting [4] (PP) or as-rigid-as-possible (ARAP) image registration [8]. Then, those transferred labels can be used by LazyBrush [26] to decompose the next frame into multiple layers. Both PP and ARAP work well for label propagation 

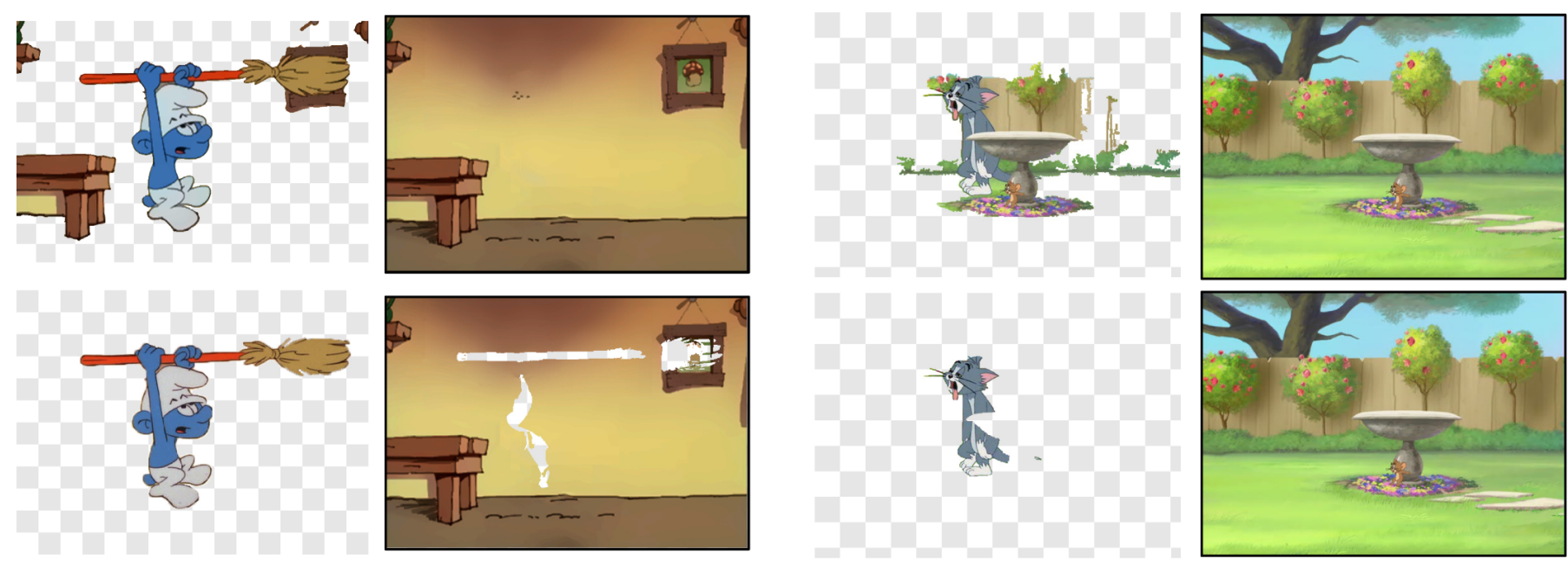

Fig. 10: Our EXCOL approach outperforms the previous cartoon processing techniques for both layer extraction and completion. Top Row: results with CBW approach [4]. Bottom Row: results with VCA approach [5]. The corresponding results with EXCOL are in Figures 1 and 9 , respectively.
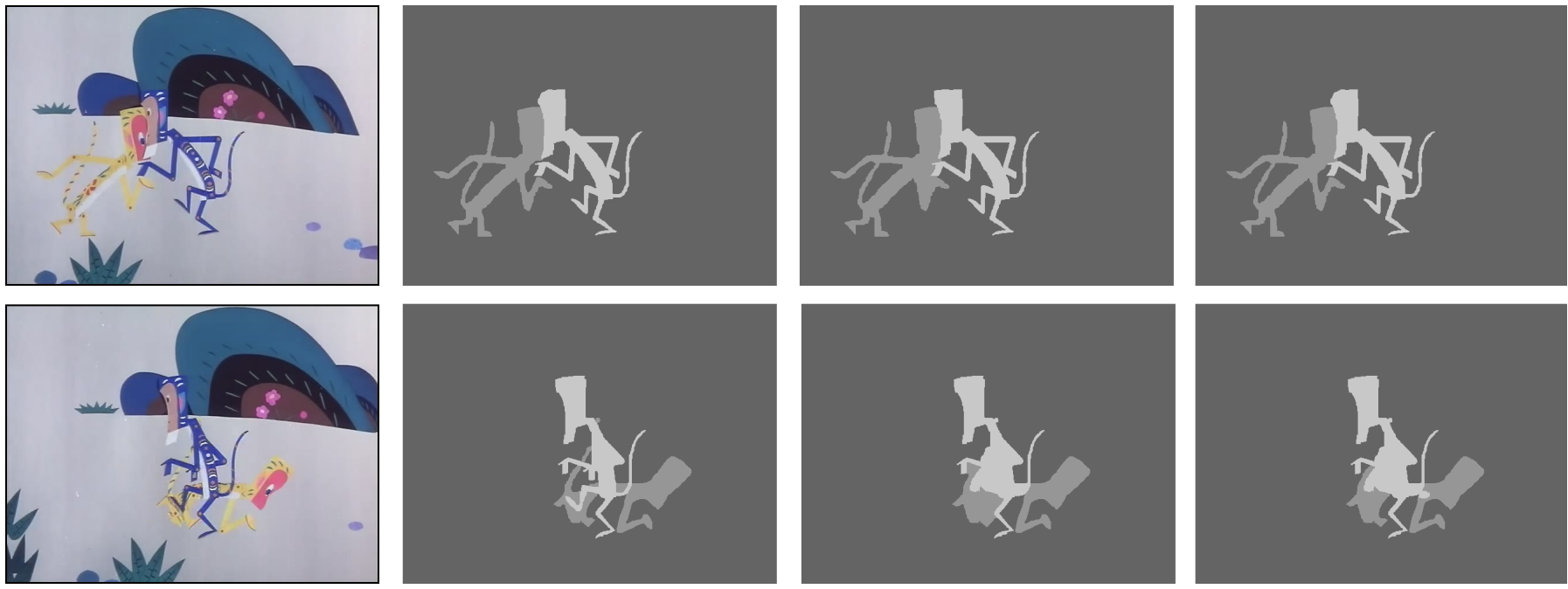

Fig. 12: Our EXCOL approach can extract correct layers compared to label propagation approaches based on local structure similarity. 1st Column: Frames of input cartoons. 2nd Column: Results with EXCOL . 3rd Column: Results with PP [4] and LazyBrush [26]. 4th Column: Results with ARAP [8] and LazyBrush [26].

on cartoons varying with slight free-form deformation between frames (e.g., examples in Figure 11 and Figure 12 (1st row)). However, unlike EXCOL treating decorative lines as compact cartoon elements, LazyBrush locates the segment boundaries at the pixels with minimal intensity in the decorative lines, which might split the decorative lines and cause artifacts on the background layer (see Figure 11). Moreover, in the case of sever occlusion (Figure 12 (2nd row)), PP and ARAP deliver labels to incorrect regions and result in mislabeled layer extraction. In contrast, our EXCOL achieves better layer extraction due to the global optimization on the similarity prediction.

To illustrate the cartoon-oriented merit of EXCOL , we have also compared our approach with some representative approaches designed for layer extraction and video completion of natural videos. In Figure 13, we compare the performance of EXCOL with layer extraction methods of bi-layer segmentation [13], time-sequential graph restriction (TSGR) [6] and multi-label propagation (MLP) approach [15]. Clearly EXCOL achieves the best layer extraction. For the two examples, bi-layer segmentation could not obtain the full representation of each layer due to the improper motion likelihood analysis on cartoons. TSGR also fails in extracting accurate layers since it is built on parametric affine motion style, which is not suitable for these two examples. MLP assigns different regions with close color to the same layer, since it only uses color information for label propagation. For example, the Smurf's red tongue is classified to the layer with red broom. In contrast, our EXCOL takes both shape and color cues into account, and is able to generate desirable layers.

EXCOL outperforms traditional video completion approaches when filling the missing regions in cartoon animation sequence. In Figure 14, we compare our completion approach with a completion approach based on tracking and fragment merging [22]. Like other video completion approaches proposed for processing general types of videos, the approach 

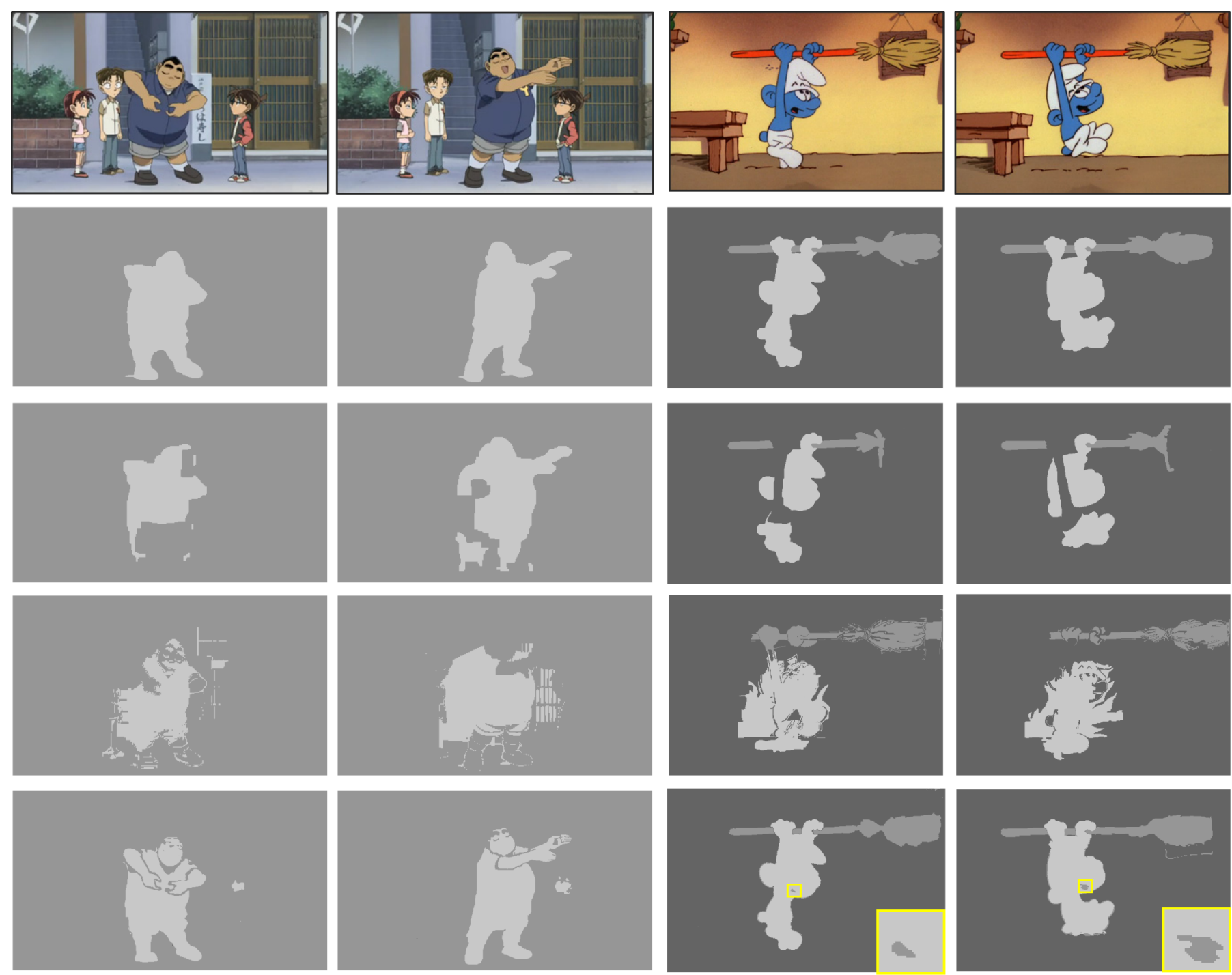

Fig. 13: Considering the unique characteristics of cartoons, our EXCOL approach produces more accurate extraction results than existing layer extraction techniques designed for natural videos. 1st Row: Frames of input cartoons. 2nd Row: Results with EXCOL . 3rd Row: Results with bi-layer segmentation approach [13]. The right example is achieved by applying the approach of [13] iteratively. 4th Row: Results with TSGR [6]. 5st Row: Results with MLP [15]. For fairness, all the results are obtained with the same initial layer labeling in one key frame.

in [22] works at the pixel level and thus works well only for small holes. Thus such approaches often cause serious artifacts in the completed results. Please see the accompanying video for the advantage of EXCOL over [22] for video completion.

Limitations. EXCOL only takes care of animated cartoons with fixed number of layers in the input video. In the case of cartoons with varied numbers of layers, we have to manually divide an input cartoon video into short clips, each of which has fixed numbers of layers. Currently, even with Laplacian diffusion to smooth the decorative lines, our layer extraction step might still generate jaggy layer boundaries, especially for hair or whiskers of characters and cartoons of low resolution. In this case, we would exploit image matting and anti-aliased rendering techniques to further improve the layer boundary quality. Our layer completion step assumes that layer order keeps fixed over time. If the occlusion order changes, the automatic layer completion step will get stuck because we cannot correctly infer the occlusion mask $\Omega$. In addition, EXCOL is established on the assumption that animation undergoes smooth transition between consecutive frames, which is common for many cartoons. However, if sharp changes occur, EXCOL might produce incorrect layer extraction and completion results. Figure 15 shows an imperfect layering example: due to the sharp change of Ponyo's shape between the first and second frames but the color similarity between her cloth regions and the girl's eye regions, the tint region on Ponyo's cloth is assigned an incorrect label. In all these cases, we have to add more interaction to improve the layering performance.

\subsection{Applications}

The layering representation can benefit many applications, e.g., motion retargeting [1] and cartoon inbetweening [2]. We have implemented four applications, namely cartoon re- 

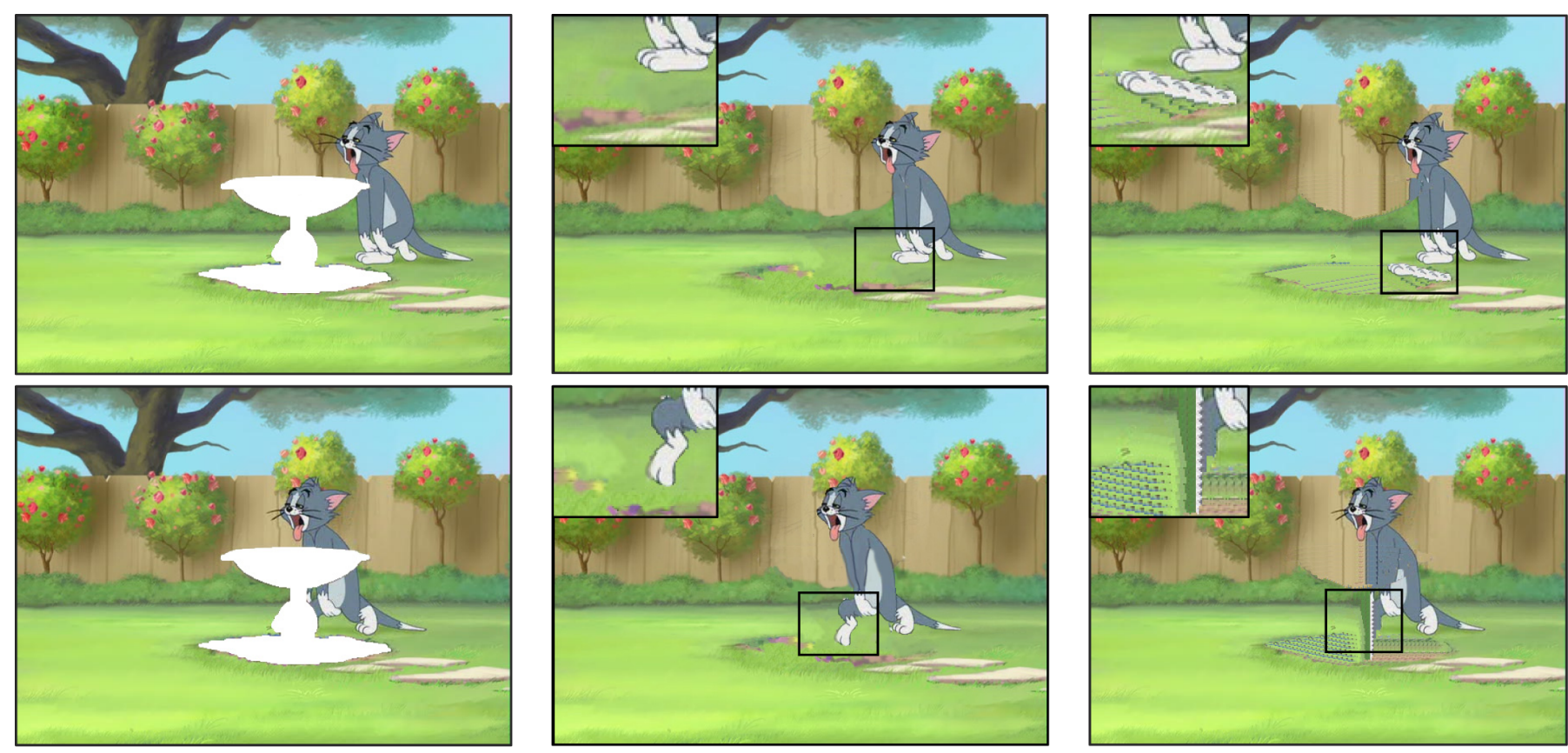

Fig. 14: Left Column: "Hole" masks (i.e., the missing fountain) need to be filled in the animation sequences. Middle Column: "Holes" are filled using our EXCOL . Right Column: "Holes" are filled using the video completion approach based on tracking and fragment merging [22]. Please refer to the accompanying video for the comparisons of the completed animation. (Cartoon images are from Tom and Jerry.)
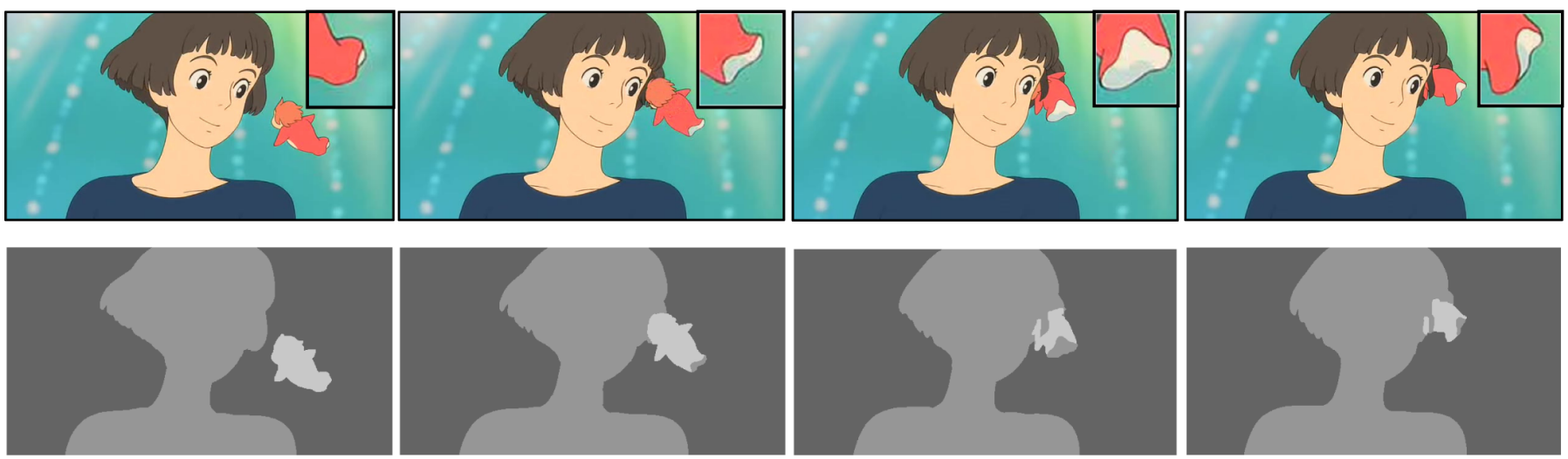

Fig. 15: EXCOL might generate imperfect layer extraction during label propagation. 1st Column: initial layering by user's guidance labeling. 2nd-4th Columns: label propagation to the next three consecutive frames. Due to the sharp change between the first and second frame, some regions on Ponyo's cloth are labeled the same as the girl. (Cartoon images are from Ponyo On The Cliff By The Sea.)

coloring, layer reshuffling, layer replacement, and cartoon composition.

Cartoon Recoloring. We can easily change the color of interested regions over the frames by extracting the layer that contains these regions. Here, it is assumed the color distribution on this layer is monotone. Then by changing the colors in the key frame, the corresponding regions in the other frames will be recolored in accord with the change in the key frame (Figure 1).

Layer Reshuffling. Our EXCOL layering approach can generate layers with complete representation, which can be used to reshuffle the characters to produce new cartoon animation. Figure 16(a) shows an example that changes the layer order and positions of characters. It is seen that finally the girl appears in front as well as her position is relocated.
Layer Replacement. Based on the layer extraction results, we can easily substitute one layer with another layer to create new animation. Figure 16(b) gives an example substituting one layer with another image through the frames. The new image layer is deformed to the shape of character layer in the original animation. It can be seen that the new layer is blended into the cartoons in a plausible way.

Cartoon Composition. The layering results can also be used to produce new cartoons by combining layers from different animations. Since all the layers have complete representations, it is easy to superimpose them seamlessly. Figure 16(c) shows a new cartoon animation that is composed of three character layers and one background layer from four animations. 

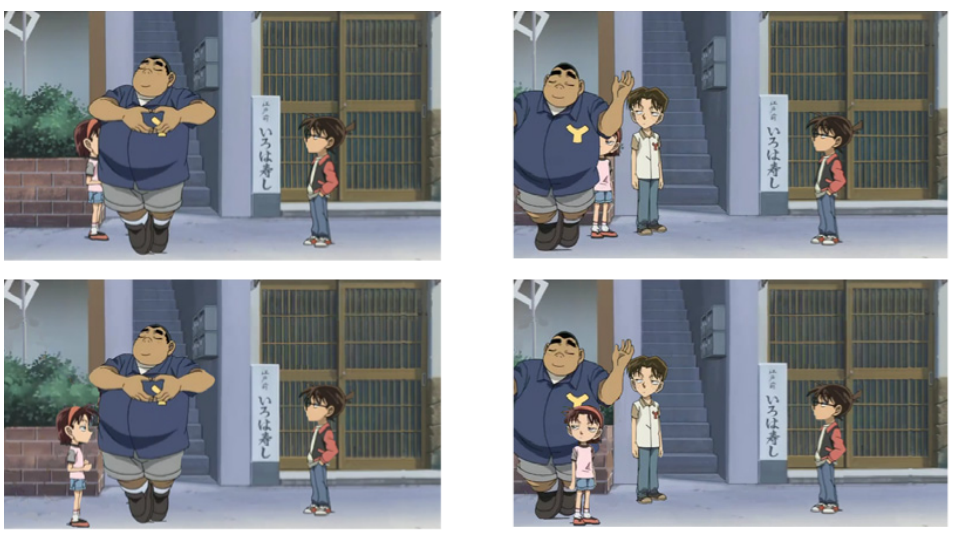

(a)
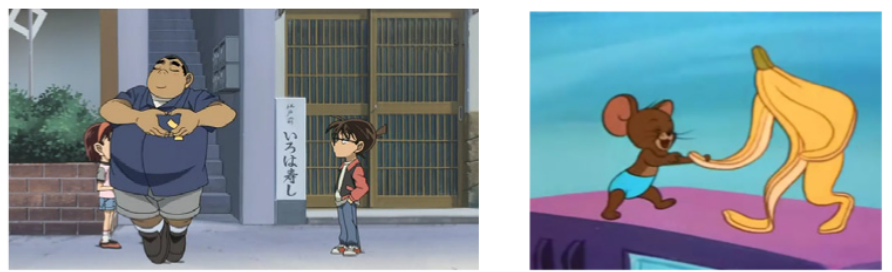
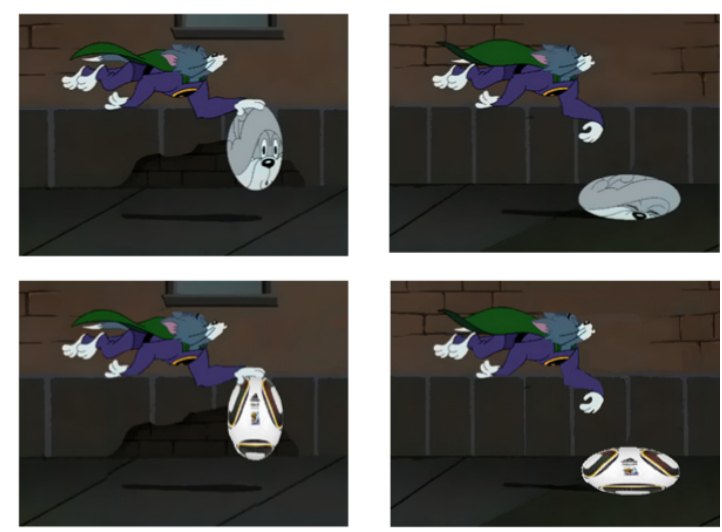

(b)
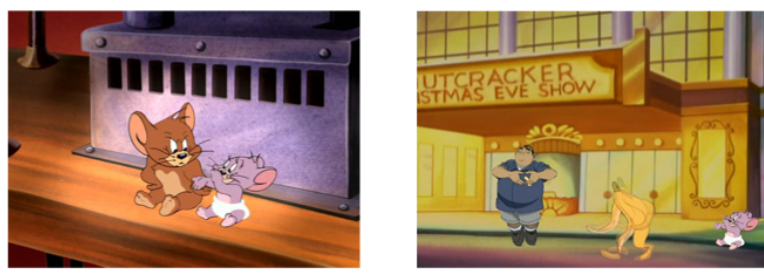

(c)

Fig. 16: The EXCOL layering results can be applied in various cartoon processing applications: (a) layer reshuffling; (b) layer replacement; (c) cartoon composition.

\section{Conclusion}

We have introduced EXCOL, a novel cartoon animation layering technique. We design layer extraction and completion methods tailored for cartoon animation by considering its unique characteristics, especially shape cues. Our technique allows reliable propagation of labels from a key frame to the rest of the animation and thus effective extraction of semantically meaningful layers. The warping-based completion is robust for large missing areas and is able to recover structural features, resulting the full-formed layer representation.

Our current implementation only deals with global layering, i.e., each layer corresponds to either a character or background, but there is also local layering in the interior of a character, which should be addressed for more practical applications. For the future work, we will extend our layering approach in a multi-level way that can extract and complete both global layers and local layers.

\section{ACKNOWLEDGMENT}

We would like to thank the anonymous reviewers for their valuable comments. This work was supported in part by grants from the National Natural Science Foundation of China (No. 60972142), the MOE-Intel Joint Research Fund (No. MOEINTEL-09-07), China Postdoctoral Science Foundation (No. 20100471618), Program for New Century Excellent Talents in University (No. NCET-09-0635), the Hong Kong Research Grant Council (No. GRF9041562, CityU113711), and the City University of Hong Kong (No. 7002533).

\section{REFERENCES}

[1] C. Bregler, L. Loeb, E. Chuang, and H. Deshpande, "Turning to the masters: Motion capturing cartoons," ACM Transactions on Graphics, vol. 21 , no. 3, pp. 399-407, 2002.
[2] C. de Juan and B. Bodenheimer, "Re-using traditional animation: Methods for semi-automatic segmentation and inbetweening," in Proceedings of Symposium on Computer Animation. Eurographics Association, 2006, pp. 223-232.

[3] D. Sýkora, J. Buriánek, and J. Zára, "Sketching cartoons by example," in Eurographics Workshop on Sketch-Based Interfaces and Modeling (2005), 2005.

[4] D. Sýkora, J. Buriánek, and J. Žára, "Colorization of black-and-white cartoons," Image and Vision Computing, vol. 23, no. 9, pp. 767-852, 2005.

[5] S. H. Zhang, T. Chen, Y. F. Zhang, S. M. Hu, and R. R. Martin, "Vectorizing cartoon animations," IEEE Transactions on Visualization and Computer Graphics, vol. 15, no. 4, pp. 618-629, May 2009.

[6] M. Fradet, P. Pérez, and P. Robert, "Time-sequential extraction of motion layers," in Proceedings of International Conference on Image Processing, 2008, pp. 3224-3227.

[7] J. Wang, Y. Q. Xu, H. Y. Shum, and M. F. Cohen, "Video tooning," ACM Transactions on Graphics, vol. 23, no. 3, pp. 574-583, Aug. 2004.

[8] D. Sýkora, J. Dingliana, and S. Collins, "As-rigid-as-possible image registration for hand-drawn cartoon animations," in Proceedings of NonPhotorealistic Animation and Rendering, 2009, pp. 25-33.

[9] C. de Juan and B. Bodenheimer, "Cartoon textures," in SCA '04, 2004.

[10] J. J. Xiao and M. Shah, "Motion layer extraction in the presence of occlusion using graph cuts," IEEE Transactions on Pattern Analysis and Machine Intelligence, vol. 27, pp. 1644-1659, 2005.

[11] J. Wang and E. Adelson, "Representing moving images with layers," IEEE Transactions on Image Processing, vol. 3, no. 5, pp. 625-638, 1994.

[12] Q. F. Ke and T. Kanade, "A robust subspace approach to layer extraction," in Proceedings of the Workshop on Motion and Video Computing. IEEE Computer Society, 2002, p. 37.

[13] A. B. A. Criminisi, G. Cross and V. Kolmogorov, "Bilayer segmentation of live video," in International Conference on Computer Vision. IEEE Computer Society, 2006, pp. 53-60.

[14] T. H. Wang, J. Collomosse, D. Slatter, P. Cheatle, and D. Greig, "Video stylization for digital ambient displays of home movies," in Syposium on Non-Photorealistic Animation and Rendering, 2010, pp. 3005-3008.

[15] T. H. Wang, J. Y. Guillemaut, and J. Collomosse, "Multi-label propagation for coherent video segmentation and artistic stylization," in International Conference on Image Processing, 2010, pp. 137-146.

[16] J. Wang, P. Bhat, R. A. Colburn, M. Agrawala, and M. F. Cohen, "Interactive video cutout," ACM Trans. Graph., vol. 24, no. 3, pp. 585594, 2005. 
[17] Y. Li, J. Sun, and H.-Y. Shum, "Video object cut and paste," ACM Trans. Graph., vol. 24, no. 3, pp. 595-600, 2005.

[18] X. Bai, J. Wang, D. Simons, and G. Saprio, "Video snapcut: Robust video object cutout using localized classifiers," ACM Transactions on Graphics, vol. 28, no. 3, 2009.

[19] J. McCann and N. Pollard, "Local layering," ACM Transactions on Graphics, vol. 28, no. 3, pp. 1-7, Aug. 2009.

[20] Y. Wexler, E. Shechtman, and M. Irani, "Space-time video completion," in Computer Vision and Pattern Recognition, 2004, pp. 120-127.

[21] I. Drori, D. Cohen-Or, and H. Yeshurun, "Fragment-based image completion," ACM Transactions on Graphics, vol. 22, no. 3, pp. 303-312, 2003.

[22] Y. T. Jia, S. M. Hu, and R. R. Martin, "Video completion using tracking and fragment merging," The Visual Computer, vol. 21, no. 8, p. 601, 2005.

[23] J. Y. Jia, T. P. Wu, Y. W. Tai, and C. K. Tang, "Video repairing: Inference of foreground and background under severe occlusion," IEEE Transactions on Pattern Analysis and Machine Intelligence, vol. 28, no. 5, pp. 832-839, 2006.

[24] Y. Li, J. Sun, C.-K. Tang, and H.-Y. Shum, "Lazy snapping," ACM Trans. Graph., vol. 23, no. 3, pp. 303-308, 2004.

[25] C. Rother, A. Blake, and V. Kolmogorov, "Grabcut - interactive foreground extraction using iterated graph cuts," ACM Transactions on Graphics, vol. 24, no. 3, pp. 309-314, 2004.

[26] D. Sýkora, J. Dingliana, and S. Collins, "Lazybrush: Flexible painting tool for hand-drawn cartoons," Computer Graphics Forum, vol. 28, no. 2, pp. 599-608, 2009.

[27] Y. Boykov and G. Funka-Lea, "Graph cuts and efficient n-d image segmentation," International Journal of Computer Vision, vol. 70, no. 2, pp. 109-131, 2006

[28] S. Belongie, J. Malik, and J. Puzicha, "Shape matching and object recognition using shape contexts," IEEE Transactions on Pattern Analysis and Machine Intelligence, vol. 24, no. 24, pp. 509-522, 2002.

[29] V. Kolmogorov and R. Zabih, "What energy functions can be minimized via graph cuts," IEEE Transactions on Pattern Analysis and Machine Intelligence, vol. 26, no. 2, pp. 147-159, Feb. 2004.

[30] Y. Boykov, O. Veksler, and R. Zabih, "Fast approximate energy minimization via graph cuts," in International Conference on Computer Vision. IEEE Computer Society, 1999, pp. 377-384.

[31] B. Zitová and J. Flusser, "Image registration methods: a survey," Image and Vision Computing, vol. 21, pp. 977-1000, 2003.

[32] D. G. Lowe, "Object recognition from local scale-invariant features," in International Conference on Computer Vision. IEEE Computer Society, 1999, pp. 1150-1157.

[33] M. A. Fischler and R. C. Bolles, "Random sample consensus: A paradigm for model fitting with application to image analysis and automated catrography," Communications of ACM, vol. 24, pp. 381395, 1981.

[34] D. Sýkora, D. Sedláček, J. C. Sun, J. Dingliana, and S. Collins, "Adding depth to cartoons using sparse depth (in)equalities," in Eurographics 2010, 2010

[35] P. Besl and N. McKay, "A method for registration of 3-d shapes." IEEE Transactions on Pattern Analysis and Machine Intelligence, vol. 14, no. 2, pp. 239-256, 1992.

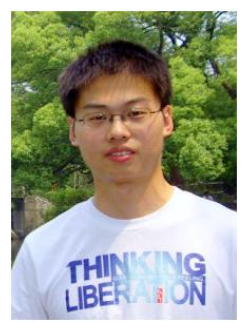

Lei Zhang received his B.S. and Ph.D. degrees in Applied Mathematics from Zhejiang University in 2004 and 2009 respectively. His research interests include computer graphics, image and video processing.

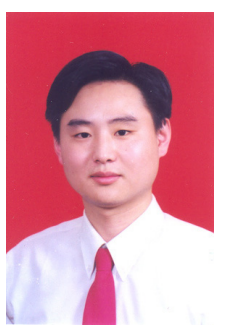

Hua Huang received his B.S. and Ph.D. degrees from Xi'an Jiaotong University in 1996 and 2006, respectively. He is currently a professor in School of Electronics and Information Engineering, Xi'an Jiaotong University. His main research interests include image and video processing, pattern recognition and machine learning. $\mathrm{He}$ is a member of the IEEE.

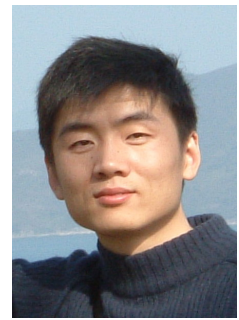

Hongbo Fu is currently an Assistant Professor in the School of Creative Media, City University of Hong Kong. Before joining CityU, he had postdoctoral research trainings at the Imager Lab, University of British Columbia, Canada and the Department of Computer Graphics, Max-PlanckInstitut Informatik, Germany. He received the $\mathrm{PhD}$ degree in computer science from the Hong Kong University of Science and Technology in 2007 and the BS degree in information sciences from Peking University, China, in 2002. His primary research interests fall in the field of computer graphics with an emphasis on digital geometry processing. 